\title{
Results of Early Mobilization and Discharge after Myocardial Infarction
}

\author{
HELEN H. TUCKER， P. H. M. CARSON， N. M. BASS， G. P. SHARRATT， J. P. P. STOCK
}

British Medical fournal, 1973, 1, 10-13

\begin{abstract}
Summary
A total of 342 patients with acute myocardial infarction who were admitted to a coronary care unit are reviewed to assess the results of early mobilization and discharge. The mean duration of admission was 8.4 days and $89 \%$ of the survivors were discharged from hospital by the tenth day. The inpatient mortality was $15.5 \%$. An additional $6.7 \%$ died during the six weeks' follow-up period, giving a total mortality of $22 \cdot 2 \%$. Altogether, $7.6 \%$ of patients were readmitted. Venous thromboembolic phenomena occurred in $3.5 \%$ during the inpatient period. Of patients who were eligible $62 \%$ were back at work five months after their myocardial infarction. We think the results justify a short hospital admission period for acute myocardial infarction.
\end{abstract}

\section{Introduction}

Price (1946) advised 'at least three months' complete bed rest . . . followed by a similar period of partial rest" in the treatment of acute myocardial infarction. Since that time there has been a progressive trend towards a shorter hospital admission period after acute myocardial infarction. Wood (1956) recommended a month in bed and a further fortnight resting at home. Friedberg (1966) suggested six weeks in bed for transmural myocardial infarction. Royston (1972) was able to discharge $40 \%$ of his patients within a fortnight of admission, and Boyle and his colleagues (1972) discharged $62 \%$ on or before the tenth day. We now report the results of the shortest hospital admission period of which we are aware.

\section{Patients and Methods}

The coronary care unit of the North Staffordshire Hospital Group contains 20 beu's, six of which are equipped for continuous E.C.G. monitoring. Only male patients are admitted and the sole criterion for admission is suspected myocardial infarction. Patients are monitored for 72 hours by using lead $\mathrm{MCL}_{1}$ (Marriott and Fogg, 1970), and for the first 24 hours they are restricted to bed on a fluid diet. After 24 hours they are allowed to sit in a chair provided they are free from significant chest pain and are not hypotensive. After the monitored period they move down the ward but remain in the unit throughout their stay in hospital. They are restricted to sitting in a chair until the sixth day when they are encouraged to walk around the ward. Between the seventh and tenth days most patients are discharged home. They are instructed to remain in their homes for one month, the first two weeks on the same level, and during the second two weeks they are permitted to go up and down stairs. Sexual intercourse is not advised until three weeks after discharge. After one month they are encouraged to start taking short walks, gradually increasing the amount of daily exercise.

\footnotetext{
Department of Cardiology, North Staffordshire Hospital Group, Stoke-on-Trent

HELEN H. TUCKER, M.B., B.s., Honorary Research Fellow

P. H. M. CARSON, B.M., M.R.C.P., Consultant Cardiologist

N. M. BASS, B.M., M.R.C.P., Honorary Senior Registrar in Cardiology

G. P. SHARRATT, M.B., M.R.C.P., Registrar in Cardiology

J. P. P. STOCK, M.D., F.R.C.P., Consultant Cardiologist
}

Six weeks after discharge they are reviewed in the outpatient clinic and are then advised to return to work unless there are definite contraindications. This is a flexible system and can be adapted to the individual. Throughout their hospital stay and follow-up period each patient is under the care of the same cardiologist.

A 12-lead E.C.G. is taken, and serum glutamic-oxaloacetic acid transaminase and serum lactic dehydrogenase are estimated daily for the first three days. A portable chest $x$-ray picture, with the patient in bed and a film-tube distance of 4-5 ft (120$150 \mathrm{~cm}$ ), is taken within 24 hours of admission and subsequently if indicated.

The management outlined above has been the practice at this hospital centre for the past five years. We now report the results of 342 men with proved myocardial infarction who were admitted between 14 December 1970 and 5 November 1971. The age range was from 32-81 years with a mean of 56.5 years. Altogether, $73.4 \%$ of patients had transmural myocardial infarction as defined by the development of pathological $Q$ waves, and $23.7 \%$ had non-transmural myocardial infarctionthat is, no new pathological $Q$ waves developed and the diagnosis was based on serial E.C.G. changes or raised levels of serum glutamic-oxaloacetic acid transaminase or lactic dehydrogenase. The extent of the myocardial infarction could not be determined in $2.9 \%$ because of persistent left bundle-branch block or death before E.C.G. changes were fully developed. Of the infarcts $55.1 \%$ were anterior or lateral, $32.2 \%$ were inferior, $0.9 \%$ were strict posterior, and other sites or combined sites were present in $5 \% ; 4.1 \%$ could not be classified.

Half of the patients were admitted within four hours of the onset of symptoms and $80 \%$ within 12 hours. A total of $23.7 \%$ had one or more previous myocardial infarcts, $44 \%$ gave a history of angina for more than two weeks before admission, and $49 \%$ had a diastolic blood pressure exceeding $95 \mathrm{~mm} \mathrm{Hg}$ at some time, either in the past or on admission or at the first follow-up visit six weeks after discharge.

Anticoagulants are used only for thrombo-embolic complications. Ventricular extrasystoles are treated with lignocaine if they meet one of the following criteria: five or more a minute, two or more consecutively, multifocal or multiform, or occurring near the peak of the preceding $T$ wave (Lown et al., 1967). If they are not suppressed by lignocaine other drugs are usedfor example, procainamide, phenytoin sodium, or practolol. Heart failure and cardiogenic shock are treated with routine anti-failure drugs. Complete heart block is treated with isoprenaline if the ventricular rate is less than 60 a minute; electrical pacing was used in only two patients.

\section{Results}

Duration of Admission. - The mean duration of admission was 8.4 days with a range of 3-29 days. The patients who were discharged before the sixth day were thought to have sustained a myocardial infarct some days before their admission to hospital. Only $9 \cdot 1 \%$ (31 patients) were kept in hospital beyond 10 days because nine patients had heart failure, five had pulmonary embolism or deep venous thrombosis, and four were kept in for social reasons. In the remaining 13 patients there was a variety of reasons such as chest infection, extension of myocardial infarction, and cerebrovascular accidents. If the home circumstances were sufficiently inadequate that a patient could not be properly cared for arrangements were made with a convalescent home and mobilization there was along similar lines 
to our usual recommendations. Inadequate home circumstances were often anticipated soon after the patient's admission and this enabled the medical social worker to make appropriate arrangements so that the patient's stay in hospital was frequently not prolonged.

Readmissions within Six Weeks of Discharge.-Altogether, $7 \cdot 6 \%$ (26 patients) were readmitted to hospital: 11 patients with further myocardial infarction, four with angina, four with pulmonary embolism, three with heart failure without further infarction, two with non-cardiac chest pain, one with Dressler's syndrome, and one with a stroke. The time of readmission ranged from 1-34 days from discharge, most being readmitted within the first two weeks.

Inpatient Mortality.-The mortality for the initial admission to the coronary care unit was $15.5 \%$ (53 patients). Of these, $40 \%$ (22 patients) died within the first 24 hours, and $2 \%$ (four patients) died after the tenth day, all with heart failure as the primary cause. The precise cause of inpatient death was often difficult to determine because the various complications are not mutually exclusive. Forty-three per cent. (23 patients) died with heart failure as the probable major cause, $32 \%$ (17 patients) with complete heart block, 19\% (10 patients) with cardiogenic shock, and $6 \%$ (three patients) died suddenly when off the monitor. Twenty-five per cent. (13 patients) died from ventricular fibrillation; in 11 of these the ventricular fibrillation was thought to be secondary to severe heart failure or cardiogenic shock and in two the arrhythmia was thought to be primary. A total of 34 necropsies were performed. Rupture of the free wall of the left ventricle was found in $14.7 \%$ of these (five patients). In three of the five patients with ruptured hearts death had occurred within 24 hours, in one on the sixth day, and in one on the tenth day.

Outpatient Mortality in Six Weeks after Discharge.-After discharge from the coronary care unit a further $6.7 \%(23$ patients) died within the next six weeks. Seven of these patients were readmitted to hospital, six to the coronary care unit. The mean time of death after discharge was 16 days with a range of 3-42 days. Of these 23 deaths 17 occurred within three weeks after discharge.

Total Mortality.-The combined mortality of inpatients and of outpatients within six weeks after discharge from hospital was $22 \cdot 2 \%$. The mean age of the patients who died was $60 \cdot 1$ years, which is significantly higher than the mean age of the total group $(P<0.001)$. A history of previous myocardial infarction was present in $27 \%$ of the patients who died, a figure not significantly different from the incidence of previous myocardial infarction in the whole group $(P<0.05)$. In the patients who died the recent myocardial infarct was transmural in $75 \%$, a figure not significantly different from the incidence of transmural infarcts in the total group $(73.4 \%)$. Transmural anterior or lateral infarction was significantly more common in patients who died $(54 \%)$ than in those who survived $(35 \%)$, $(P<0.005)$. Transmural inferior myocardial infarction was significantly less in patients who died $(17 \%)$ than in those who survived $(32 \%)(P<0.025)$. The mean time from the onset of symptoms to admission in the patients who died was 5 hours and in the total group was 5.9 hours; this difference is not significant $(P>0.05)$. Heart failure was present during admission in $72 \%$ of the patients who died and in $34 \%$ of the survivors; this difference is significant $(P<0.001)$. The mortality in patients with a cardio-thoracic ratio exceeding $59 \%$ in any film taken during the acute admission period was $43 \%$, whereas when the cardio-thoracic ratio was less than $60 \%$ the mortality was $13.5 \%$. This difference is highly significant $(P<0.001)$.

\section{COMPLICATIONS}

\section{Cardiogenic shock}

Shock not due to arrhythmias was present in 18 patients on admission and occurred in a further 14 during admission. Thus shock occurred in $9.1 \%$ (32 patients). Twenty-seven of these patients died during admission, a mortality of $84 \%$. Two of the five patients who had shock and were discharged from hospital died before their six-week follow-up visit.

\section{Arrhythmias}

Complete heart block occurred in $9 \%$ (28 patients). Of these, $64 \%$ died in hospital (18 patients). Thirteen of the patients with complete heart block had anterior myocardial infarcts, and had a mortality of $78 \%$. Eleven had inferior myocardial infarcts and their mortality was $36 \%$. In four patients the sites of their myocardial infarcts could not be determined and all these patients died. All who survived episodes of complete heart block returned to sinus rhythm and only one of the 10 survivors died in the first six weeks after discharge from hospital.

Ventricular extrasystoles fulfilling the criteria for attempted suppression occurred in $17 \%$ (59 patients). Twenty-seven per cent of these patients died in hospital and a further $8.5 \%$ died after discharge. The inpatient mortality is significantly higher than that of the total group $(P<0.01)$.

Ventricular tachycardia occurred in $4 \%$ (14 patients) and the inpatient mortality was $50 \%$. No patients died after discharge.

Ventricular fibrillation occurred in 4\% (14 patients). In 11 patients the arrhythmia was thought to be secondary to cardiogenic shock or heart failure and all these patients died. Three had primary ventricular fibrillation and two of these died.

Altogether, $8.5 \%$ (29 patients) developed atrial fibrillation. In over half this arrhythmia was present on admission and in only five patients did this arrhythmia occur after the fifth day. Thirty-five per cent. (10 patients) died in hospital and one patient after discharge; this is a significantly higher mortality than in the whole group $(P<0.01)$. All survivors were in sinus rhythm when they left hospital.

Sinus bradycardia occurred in $11 \%$ (39 patients). In 23 patients the arrhythmia was present on admission. Twenty-six per cent. (10 patients) died in hospital and two after discharge from hospital. Although the mortality is higher than in the whole group the difference is not significant at the 5\% level. In 19 patients the myocardial infarct was inferior, in 16 anterior or lateral, and in four patients the site could not be determined. Twenty per cent. (eight patients) of the 39 patients with sinus bradycardia developed complete heart block, which is significantly higher than in the whole group $(P<0.01)$.

\section{Thromboembolic Complications}

Clinical evidence of deep vein thrombosis occurred in $2 \%$ (seven patients) during admission. None developed symptoms or signs suggestive of deep venous thrombosis after discharge from hospital.

Clinical or radiological evidence of pulmonary embolism occurred in $1.8 \%$ (six inpatients). A further five were thought to have had pulmonary embolism after discharge from hospital and four of these were readmitted. Thus 12 inpatients and five outpatients had clinical evidence of venous thromboembolic phenomena, which is $5 \%$ of the total group. In only one patient were both deep vein thrombosis and pulmonary embolism clinically evident.

Cerebrovascular accidents occurred in four patients during admission and in two patients after discharge from hospital. Evidence of peripheral vascular occlusion occurred in two patients in hospital and in none after discharge.

\section{Further Myocardial Infarction}

Evidence of further myocardial infarction during the initial admission period occurred in $2.9 \%$ (10 patients). The time of recurrence ranged from $0-8$ days and was commonest on the fourth day. Two patients died in hospital and two after discharge from hospital. Of $3.2 \%$ (11 patients) who were readmitted with a further myocardial infarction, five died. 


\section{Heart Failure}

Heart failure was thought to be present in $50 \%$ (171 patients) during admission. The diagnosis is based on clinical evidence alone in 85 patients, on radiological features alone in 30, and on combined clinical and radiological evidence in 56. In 26 patients there were symptoms or signs suggestive of heart failure at six weeks and 17 of these had had heart failure during admission. Chest $x$-ray pictures were taken in 165 patients at the six-weeks follow-up visit. Of these, $4 \cdot 25 \%$ had a cardio-thoracic ratio exceeding $60 \%$.

\section{RETURN TO WORK}

Of the 342 patients admitted to hospital, 205 have now been seen at the second follow-up visit three months after their first onethat is, about five months after their admission. Forty-one of these men had retired before their myocardial infarcts, leaving 164 who were eligible for work. Of the latter group, $62 \%$ were back at work and $94 \%$ of these were back at the work which they were doing before their myocardial infarction.

\section{Discussion}

In this hospital centre the introduction of a coronary care unit reduced the mortality after admission for acute myocardial infarction from $30 \%$ to $18 \%$ in the first year (J.P.P.S. Stock, personal communication, 1969). Similar results have been reported from many centres. The short hospital admission period and the early mobilization which are discussed in this paper have been the practice of our coronary care unit for the last five years.

The mortality in coronary care units is affected greatly by the speed with which patients are admitted after the onset of symptoms. When this is prolonged some of the patients at high risk will have died before admission and the consequent hospital mortality will be low. When it is short, hospital mortality will be greater. In this series the median time from the onset of symptoms to admission was four hours, a figure comparable to that reported from several other centres (Pentecost and Mayne, 1968; Gilchrist, 1971; Smyllie et al., 1972).

Our initial inpatient mortality was $15.5 \%$ with a mean duration of admission of 8.4 days. The total mortality six weeks from discharge was $22.2 \%$. Others have reported an inpatient mortality of $18.5 \%$ with a mean duration of admission of four weeks (Pentecost and Mayne, 1968) and an inpatient mortality of $16.2 \%$ with a mean duration of admission of 21 days (Chapman, 1971). Thompson and Sloman (1971) had a mortality of $24.5 \%$ at 24 days. Although it is of doubtful validity to make comparisons between coronary care units, our mortality seems to be comparable with that reported from other units with a longer hospital stay.

The incidence of pulmonary embolism during the inpatient period was $1.8 \%$ and for deep venous thrombosis was $2 \%$. The diagnosis was based on clinical findings and chest $x$-ray pictures. Murray et al. (1970) reported an incidence of $34 \%$ for deep venous thrombosis when isotopic methods were used and $20 \%$ when diagnosed clinically. In their series $11 \%$ were thought to have had pulmonary emboli. Maurer et al. (1971) reported an incidence for deep venous thrombosis of $34 \%$ diagnosed isotopically and $27 \%$ diagnosed clinically. In their series $9 \%$ were thought to have had pulmonary emboli. The low incidence of thromboembolic complications in this series was probably due in part to diagnostic imperfections but it seems likely that it was also due to early mobilization.

Early mobilization should lead to a more rapid return to work. This appears to be borne out by the present series where $62 \%$ of those who were eligible had returned to work between six weeks and five months after discharge from hospital. This compares favourably with other reports (Nagle et al., 1971;
Groden, 1967) but it is noteworthy that in Royston's (1972) report $23 \%$ of patients returned to work within four weeks from the onset of their myocardial infarcts.

Early mobilization and discharge have several potential adverse effects. Rupture of the free wall of the left ventricle was known to have occurred in five patients in the present series, an incidence of $14.7 \%$ of those who died and had necropsies. Since the numbers are small little weight can be placed on this but the incidence is similar to that in other larger series (Spiekerman, et al., 1962; Meurs, et al., 1970). In three of the present cases rupture occurred within the first 24 hours when the patients were still in bed. Therefore there is no evidence that early mobilization predisposes to this.

Schlichter et al. (1954) suggested that inadequate bedrest predisposed to ventricular aneurysm formation. We have insufficient data to confirm or refute this, but less than $5 \%$ of patients had a cardio-thoracic ratio exceeding $60 \%$ when they were seen six weeks after discharge from hospital; most of these patients had had heart failure and cardiomegaly during their inpatient period. It seems unlikely, therefore, that early mobilization leads to large aneurysm formation.

Readmissions to hospital are inevitable however long patients with acute myocardial infarction are kept in. The earlier the discharge the higher will be the readmission rate, and in this series $7 \cdot 6 \%$ of patients were readmitted. It seems unlikely that retention of these patients in hospital would have avoided the illnesses which necessitated their readmission.

Altogether $6.7 \%$ of this series died after discharge from hospital within the following six weeks. Most deaths occurred within three weeks after discharge from hospital, and even if these patients had remained in hospital it seems improbable that their lives would have been saved. The results of late hospital cardiac arrests are poor (Thompson and Sloman, 1971).

It is easy to monitor patients sitting out of bed by using the $\mathrm{MCL}_{1}$ lead system (Marriott and Fogg, 1970). This system helps to differentiate between supraventricular beats with aberrant conduction and ventricular ectopic beats, and is also useful in distinguishing right and left bundle-branch blocks.

High risk factors in this series have been much the same as in others. Shock is known to have a high mortality and our series was no exception. Heart failure was common, occurring in half the patients in hospital, and it was thought to be the major cause of death in $43 \%$ of inpatient deaths. It was also the most common single reason for keeping a patient in hospital beyond 10 days. We find that the diagnosis of heart failure after acute myocardial infarction can be difficult and we diagnose and treat heart failure on any one of the following features: otherwise unexplained dyspnoea, basal rales, an apical third heart sound, or radiological suggestion of pulmonary venous hypertension. We were surprised to find the apparent predictive value of the cardio-thoracic ratio measured from portable chest $x$-ray pictures. When this exceeded $59 \%$ the mortality in the acute admission period was $43 \%$, whereas it was only $13.5 \%$ when the cardio-thoracic ratio was less than $60 \%$.

Our series is comparable with that of Norris et al. (1969) in that increased age and transmural myocardial infarction are accompanied by a worse prognosis; also the mortality appeared to be unaffected significantly by previous myocardial infarcts.

Lown (1969) suggested that ventricular arrhythmias are not accompanied by a significantly increased risk. However, half of the present 14 patients with ventricular tachycardia died and nearly one-third of patients with ventricular extrasystoles that required attempted suppression died in hospital; a further $8.5 \%$ died after discharge. We therefore agree with Thompson and Sloman (1971) and Boyle et al. (1972) that persistent ventricular arrhythmias are associated with a higher mortality. In addition over one-third of patients with atrial fibrillation died in hospital, and we feel that the often serious nature of this arrhythmia that has been stressed by Lown et al. (1967) is not widely appreciated. Although Thompson and Sloman (1971) advocated more prolonged periods of monitoring and of hospital stay for those patients at greater risk, we, like Boyle et al. (1972), are 
not aware of evidence to show that prolongation of hospital stay in these patients will result in a lower mortality.

We have adopted a conservative attitude to the treatment of complete heart block and all patients were treated medically except for two who were paced electrically. Anterior infarction as a cause of heart block was associated with a higher mortality $(78 \%)$ than inferior infarction $(36 \%)$. These figures compare adequately with those of Lassers and Julian (1968) and of Scott et al. (1967) who paced much more frequently than we have done. We are not convinced, however, that our conservative approach is the correct one.

We conclude that a shorter period in hospital and more rapid mobilization than are normally practised are justified. By so doing beds in coronary care units are made available for patients who are at the highest risk within the first few hours and days after acute myocardial infarction.

We thank Mr. W. Deaville, Mr. John Silk, all the nursing staff on the coronary care unit, and Mrs. J. Christian, for their co-operation, Mrs. O. Adams and Mrs. I. Roscoe for secretarial help, Mrs. Leslie Barker of the Heart Research Fund of North Staffordshire for the purchase of monitoring and $x$-ray screening equipment, Dr. $M$. Kennedy and Dr. B. Cromie for much helpful advice, and Hoechst Pharmaceuticals for financial support.

We also thank the many senior house officers who have worked in the cardiac department for all the help they gave us, and Miss Julia $M$. Massey, of the computer department of the North Staffordshire Hospital Group, who kindly performed the statistical analysis.

\section{References}

Boyle, D. McC., Barber, J. M., Walsh, M. J., Shivalingappa, G., and Chaturvedi, N. C. (1972). Lancet, 2, 57.

Chapman, B. L. (1971). British Heart fournal, 33, 643.

Friedberg, C. K. (1966). Diseases of the Heart, 3rd edn., p. 892. Philadelphia, Saunders.

Gilchrist, I. C. (1971). British Medical fournal, 4, 153.

Groden, B. M. (1967). Scottish Medical fournal, 12, 297.

Lassers, B. W., and Julian, D. G. (1968). British Medical fournal, 2, 142.

Lown, B. (1969). Archiv für klinische Medizin, 216, 201.

Lown B. (1967). Journal of the American Medical Association, $199,188$.

the American Medical Association, 199, 188.
Lown, B. et al. (1967). American Yournal of Cardiology, 20, 494.

Marriott, H. J. L., and Fogg, E. (1970). Modern Concepts of Cardiovascular Disease, 39, 103

Maurer, B. J., Wray, R., and Shillingford, J. P. (1971). Lancet, 2, 1385.

Meurs, A. A. H., Vos, A. K., Verney, J. B., and Gerbrandy, J. (1970). British Heart fournal, 32, 232.

Murray, T. S., Lorimer, A. R., Cox, F. C., and Lawrie, T. D. V. (1970). Lancet, 2, 792.

Nagle, R., Gangola, R., and Picton-Robinson, I. (1971). Lancet, 2, 454.

Norris, R. M., Brandt, P. W. T., Caughey, D. E., Lee, A. J., and Scott, P. J. (1969). Lancet, 1, 274.

Pentecost, B. L., and Mayne, N. M. C. (1968). British Medical fournal, 1,

Price, F. W. (1946). Textbook of the Practice of Medicine, 7th edn., p. 1028. London, Oxford University Press.

Royston, G. R. (1972). British Heart fournal, 34, 526.

Schlichter, J., Hellerstein, H. K., and Katz, L. N. (1954). Medicine, 33, 43. Scott, M. E., Geddes, J. S., Patterson, G. C., Adgey, A. A. J., and Pantridge,

Smyllie, H. C., Taylor, M. P., and Cuninghame-Green, R. A. (1972). British Medical fournal, $1,34$.

Spiekerman, R. E., Brandenburg, J. T., Achor, R. W. P., and Edwards, J. E. (1962). Circulation, 25, 57

Thompson, P., and Sloman, G. (1971). British Medical fournal, 4, 136.

Wood, P. (1956). Diseases of the Heart and Circulation, 2nd edn., p. 750. London, Eyre and Spottiswoode.

\title{
Anti-oestrogen Therapy for Breast Cancer: A Trial of Tamoxifen at Two Dose Levels
}

\author{
H. W. C. WARD
}

British Medical fournal, 1973, 1, 13-14

\section{Summary}

Tamoxifen (ICI 46474) was given by mouth to patients with advanced, recurrent, or metastatic breast carcinoma. At a dosage of $10 \mathrm{mg}$ twice daily $60 \%$ of patients showed arrest or reversal of tumour growth. At a dosage of $20 \mathrm{mg}$ twice daily $77 \%$ showed arrest or reversal of tumour growth. Side effects were usually trivial and their incidence was the same at both dose levels. No patients showed virilization or fluid retention.

\section{Introduction}

Tamoxifen is the trans-isomer of 1 (p- $\beta$-dimethylaminoethoxyphenyl)-1, 2-diphenylbut-1-ene. In several but not all mammalian species it is a potent anti-oestrogen. It is thought to act by blocking oestrogen receptors. Nevertheless, very high dosage in rats has an oestrogenic effect while low dosage is antioestrogenic. The first clinical use of an anti-oestrogen for breast carcinoma was described by Kistner and Smith (1960) and further trials were described by Herbst et al. (1964). The only published report on the use of tamoxifen is that of Cole et al. (1971).

Department of Radiotherapy, Queen Elizabeth Hospital, Birmingham B15 2TH

H. W. C. WARD, M.R.C.P., F.F.R., Consultant Radiotherapist

\section{Patients and Methods}

Sixty-eight patients were admitted to the trial. They all had either very advanced primary carcinoma of the breast, recurrence on the chest wall, or soft tissue metastases which could be measured. Other metastases might have been present but these did not affect admission to the trial. In most patients a histological diagnosis was available but in a few patients with very advanced primary disease a biopsy specimen had not been obtained. No patient was admitted to the trial unless there had been a lapse of at least three months from previous treatment with hormones or cytotoxic drugs and from the menopause, either natural or artificial.

Patients were divided into four groups depending on the presence or absence of blood-borne metastases and whether more or less than five years had elapsed from the menopause. For each group a previously prepared randomization table was used to allocate a patient to low or high dose treatment so that the number receiving low or high dosage in each group would be similar. The dosage of tamoxifen (ICI 46474) was either $10 \mathrm{mg}$ or $20 \mathrm{mg}$ twice daily.

During the first six months of treatment patients were seen at least monthly, and thereafter some were allowed a two-month interval between visits. At the beginning of the trial all patients had haematological and biochemical investigations at least every month but when normal results had been obtained regularly for six months the interval was lengthened.

Symptomatic Side Effects.-The incidence of symptoms attributable to the drug is shown in table $I$. When a patient had more than one symptom each has been noted so that the number of symptoms recorded exceeds the number of patients 